\title{
Primary invasive laryngeal mycosis in an immunocompetent patient: a case report and clinico-epidemiological update
}

Supram Hosuru Subramanya ${ }^{1 *}$ D, Joseph Jillwin², Shivaprakash Mandya Rudramurthy ${ }^{2}$, Krishna Chandra Rijal ${ }^{1}$, Niranjan Nayak', Arunaloke Chakrabarti ${ }^{2}$ and Arnab Ghosh ${ }^{1}$

\begin{abstract}
Background: Laryngeal aspergillosis is uncommon and is usually secondary to pulmonary involvement in immunocompromised patients. Primary laryngeal aspergillosis in immunocompetent individuals is extremely rare, with a few cases documented over the last five decades.

Case presentation: We report a case of primary localised laryngeal aspergillosis in a 21-year-old apparently immunocompetent student. Septate hyphae were observed on histopathology of the laryngeal lesion, which was further confirmed as Aspergillus fumigatus after extraction of fungal DNA from formalin fixed paraffin embedded tissue (FFPET) and sequencing. The patient responded well to oral itraconazole therapy over a month.

Conclusions: Since last few decades, cases of primary laryngeal aspergillosis in immunocompetent individuals are on the rise, globally. This is the first case of invasive laryngeal aspergillosis reported in Nepal. The extraction of DNA from tissue and sequencing helps to identify the etiological agent, when culture fails to isolate the fungus.
\end{abstract}

Keywords: Primary laryngeal aspergillosis, Aspergillus fumigatus, FFPE-PCR

\section{Background}

Primary fungal laryngitis is commonly attributable to yeasts such as Candida, and Cryptococcus or fungi are known to cause endemic mycoses like Blastomyces, Paracoccidioides, and Coccidioides. The mold forms, such as Aspergillus and Mucor, may involve larynx as secondary pulmonary invasion $[1,2]$. Immunocompromisation due to leukaemia, AIDS, severe aplastic anaemia, lymphoreticular neoplasms, or immunosuppressive therapy predispose person to invasive fungal infection $[1,2]$. Primary laryngeal aspergillosis in immunocompetent individuals is extremely rare. It often mimics the pre-malignant and malignant conditions (squamous cell carcinoma) of larynx. Fungal laryngitis is usually characterized by sore throat, earache, hoarseness of voice, cough, odynophagia, formation of endolaryngeal and perilaryngeal white plaques, granulation tissue, ulcerations, erythema and edema [3].

\footnotetext{
* Correspondence: supram.gowda@gmail.com

${ }^{1}$ Department of Medical Microbiology, Manipal College of Medical Sciences, Pokhara, Nepal

Full list of author information is available at the end of the article
}

Diagnosis and prompt treatment are essential to prevent complications like scarring of the vocal folds, compromised airway due to glottic edema and dissemination of the pathogen. First case of aspergillosis of larynx was reported in 1969 from Pondicherry, South India [4]. Globally, less than 50 cases over the period of last 50 years have been documented. Herein, a case of primary laryngeal aspergillosis in an apparently immunocompetent young adult is reported. To the best of our knowledge, this is the first such case report from Nepal. In this endeavour, we conducted a comprehensive review of literature and analysed all previously reported cases.

\section{Case presentation}

A 21-year young male presented to Manipal Teaching Hospital, Pokhara, with progressive hoarseness of voice for two months and frequent cough with expectoration since one month. He had no history of phonotrauma, apparent immune deficiency, leukaemia, malignant disease, diabetes mellitus, broad-spectrum antibiotics or immunosuppressive therapy, including corticosteroids. He was not 
habituated to tobacco or alcohol. He did not have any previous history of laryngeal trauma, allergies or mycosis. A general physical examination did not reveal lymphadenopathy or organomegaly. There were no visible lesions or masses in the oral cavity, oropharynx or nasopharyngeal mucosa. His paranasal sinuses and chest X-rays were clear. Routine blood test report was within normal limits. Serological markers for Hepatitis B, C, and HIV were negative and VDRL test was non-reactive.

\section{Clinical examination and laboratory findings}

A direct laryngoscopic examination was performed under general anaesthesia. Videostroboscopy revealed a smooth, diffused whitish spheroid submucosal mass on the anterior surface of the left vocal cord. Vocal cord mobility was normal bilaterally, the airway was adequate, and both subglottis and supraglottis showed normal mucosa.

In order to exclude, glottic carcinoma the patient was subjected to punch biopsy from the lesion by the micro-laryngeal procedure under general anaesthesia. The histopathological examination showed conidia and broad septate hyphae, most of them showing acute angle branching without any evidence of malignant cells (Fig. 1). Repeat biopsy specimen processed for fungal culture did not yield any growth. For the identity of fungi, sections from paraffin-embedded tissue block were analysed by Polymerase Chain Reaction (PCR).

\section{Molecular identification by PCR Extraction of DNA from formalin fixed paraffin embedded (FFPE) tissue}

To avoid environmental fungal DNA (or) amplicon contamination, all steps were performed inside laminar air flow cabinets in separate closed cabins equipped with a dedicated set of micropipettes and instruments. A $50 \mu \mathrm{m}$ thick FFPE tissue section was cut using a clean blade by microtomy and transferred to a $1.5 \mathrm{ml}$ microcentrifuge tube. Deparaffinization and DNA extraction was performed as per Anna Lau et al. [5] with minor modifications where phenol- $\mathrm{CHCl}_{3}$-isoamyl alcohol extraction protocol was performed. The pellet was dissolved in $50 \mu \mathrm{m}$ of nuclease-free water and stored at $-20^{\circ} \mathrm{C}$ until further testing.

\section{Amplification of $28 \mathrm{~s}$ region of $r D N A$}

PCR was performed in a $45 \mu \mathrm{l}$ mixture consisting of $1 \times$ PCR buffer without $\mathrm{MgCl}_{2}$ (Genei, Bangalore), $2.5 \mathrm{mM}$ $\mathrm{MgCl}_{2}, 0.25 \mathrm{mM}$ deoxyribonucleotide blend (Fermentas), $0.4 \mu \mathrm{M}$ primers 12F 5'GTTGATAGAAYAATGTAGATAAGG3' and 13R 5'GACAGTAAGATTCCCCTTG3' (1) (Eurofins), $1.2 \mathrm{U}$ of Taq Polymerase (Bangalore Genie) and $5 \mu \mathrm{l}(\sim 80 \mathrm{ng})$ of template DNA. Thermal cycling was performed in an Eppendorf Mastercycler Gradient thermal cycler (Eppendorf AG, Hamburg) with the following conditions: denaturation at $95{ }^{\circ} \mathrm{C}$ for 10 mins followed by 60 cycles of $94{ }^{\circ} \mathrm{C}$ for 15 secs, annealing at $53{ }^{\circ} \mathrm{C}$ for 20 secs, and extension $72{ }^{\circ} \mathrm{C}$ for 25 secs and finally once at $72{ }^{\circ} \mathrm{C}$ for 5 mins. Positive and negative controls were included. Amplification was confirmed by electrophoresis on $2 \%$ agarose gel with ethidium bromide, and the amplicon was purified using Qiagen gel extraction kit according to manufacturer's instructions.

\section{Sequencing}

Bidirectional Sanger sequencing of purified amplicons was performed with the primers mentioned above, using the BigDye Terminator sequencing ready reaction kit $(\mathrm{v}$ 3.1) and the products was capillary electrophoresed in an ABI Prism 3130 genetic analyser (Applied Biosystems). Sequences were analysed using Bionumerics software version 7.1 (Applied Maths, Ghent, Belgium and identified through BLASTn (https://blast.ncbi.nlm.nih.gov/Blast.cgi? PAGE_TYPE=BlastSearch). On the basis of comparing the

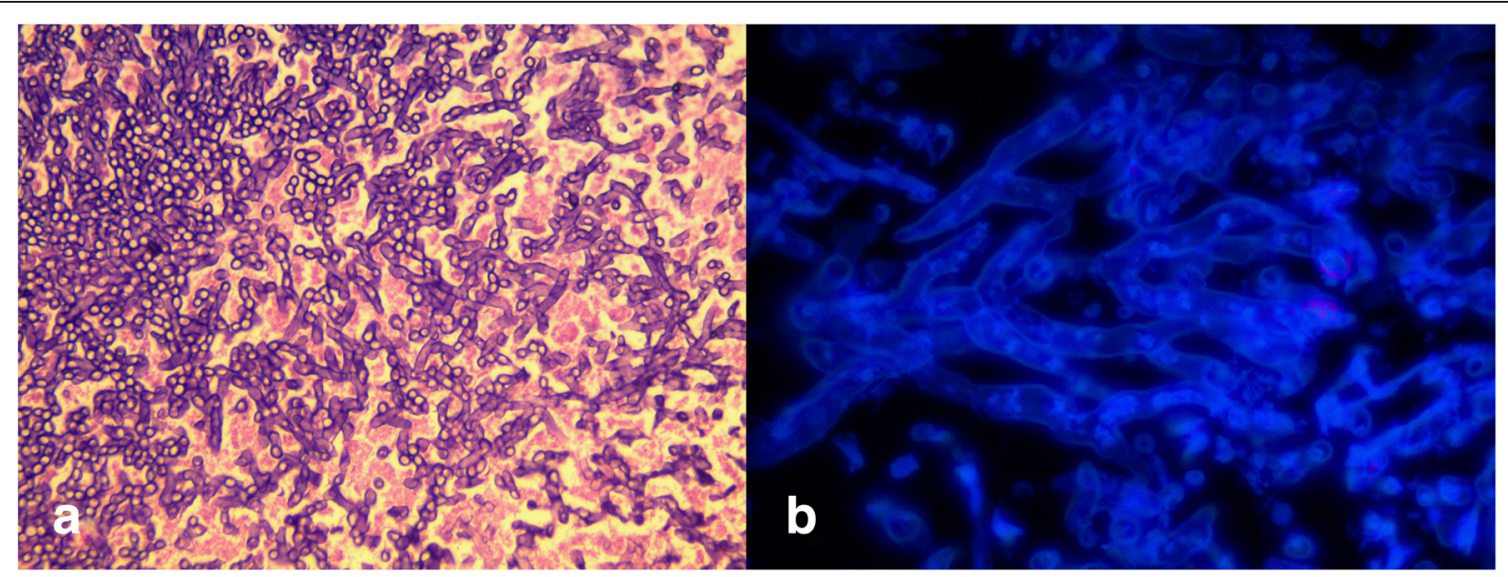

Fig. 1 a Histopathological features of biopsy sample showing numerous septate hyphae, "spaghetti-like" fungal filaments branching at an angle of approximate $45^{\circ}$, interspersed with shreds of vocal cord squamous epithelium (Haematoxylin and Eosin stained, magnification X400. b Calcofluor white staining of tissue section observed under fluorescent microscopy showing numerous branched septate hyphal forms X1000 
sequences from the specimen with those in the GenBank databases, the fungus in the biopsy was identified as Aspergillus fumigatus. Our isolate had $100 \%$ matches with the standard strain of ATCC 1022. The sequence data have been deposited in the GenBank database (http://www.ncbi.nlm.nih.gov/Genbank/index.html) with the accession number MH465665.

\section{Treatment and follow-up of the patient}

According to the clinical features and the mycological data, diagnosis of vocal cord aspergillosis was made and the patient was treated with oral itraconazole $200 \mathrm{mg}$ BD for four weeks. Clinical improvement was noticed after a month of antifungal therapy. At the third months' follow up, his voice had returned to normal, and no residual lesion was seen on laryngoscopy. At the end of 6 months follow up, there was no recurrence.

\section{Literature review}

Extensive search in PubMed/MEDLINE and Google Scholar by two investigators independently, revealed only 38 peer-reviewed reported cases of primary laryngeal aspergillosis in immunocompetent subjects (Table 1).

\section{Discussion}

\section{Aetiology of laryngeal aspergillosis}

Primary invasion of the larynx by Aspergillus is uncommon and is very rare in immunocompetent individuals. As per the literature review, till date, 38 cases of primary laryngeal aspergillosis in immunocompetent patients have been documented over 50 years. Aspergillus fumigatus was reported to be the underlying causative mould in the majority of cases, documented so far; except for two cases. A. niger infection reported by Gangopadhyay et al. from India [6] and Gallo and colleagues from Italy reported Aspergillus flavus as the etiologic agent in a patient with Felty's syndrome [7]. In the present case a immunocompetent student had $A s$ pergillus fumigatus responsible for the laryngeal pathology.

\section{Disease pathogenesis}

Aspergillus is a well-known opportunistic fungus causing allergic and invasive disease in immunocompromised hosts [8]. The pathogenesis of laryngeal aspergillosis in an immunocompetent host is not well understood. The Aspergillus conidia are ubiquitous in nature as the fungus grows in a saprophytic environment (soil and decaying matter), it could well be possible that exposure of heavy fungal load in air may allow the fungus colonise the dark airway cavities [3] which could favour their slow germination without any symptoms. Such colonisation of the paranasal sinuses leads to fungal ball formation [9]. Hoarseness of voice was the only symptom in this case without any other predisposing conditions like corticosteroid or systemic antibiotic therapy. This is in contrast with other reports where patients developed symptoms after being treated with corticosteroid or systemic antibiotic therapy or after vocal abuse for many years (Table 1).

\section{Epidemiology and risk factors for developing primary laryngeal aspergillosis}

Amongst the reviewed 38 reported cases 16/37 (44\%) were males, and 21/37 (57\%) were females. Age group ranged from 12 to 74 years. Dutta $\mathrm{M}$ et al. [3] reported in 2015 that $50 \%$ of immunocompetent subjects with primary laryngeal aspergillosis had no identifiable contributory factors, but $14.29 \%$ had vocal abuse and steroid intake. Smoking, broad-spectrum antibiotics and exposure to radiation was detected in $10.7 \%$ of cases. Of the cases, $7.1 \%$ had vocal fold cyst, whereas $3.6 \%$ had a history of COPD, oral sex and diabetes. Six cases have been reported between 2015 and 2017 (Table 1); three of these cases were without any identifiable risk factors. Remaining three cases (and a few documented prior to 2015) had a history of using corticosteroid inhalers for bronchial asthma, which could have led to abrogation of the local immunity in the throat or could have altered the flora of the laryngeal mucosa, allowing the overgrowth of Aspergillus [10]. The exact predisposing conditions contributing towards the disease progression in the present case remains obscure but could be multifactorial with a complex interplay between host and the environment.

\section{Time trend and geographical distribution of primary laryngeal aspergillosis}

Laryngeal aspergillosis in the immunocompetent individual, though infrequently reported, seems to be an emerging condition. Lack of definite guidelines for clinical diagnosis due to the rarity of the disease might have resulted in under-reporting in the past. As shown in Fig. 2, the incidence of primary laryngeal aspergillosis in immunocompetent patients has been steadily rising over the past ten years. It seems that there has been a higher rate of reporting of the cases, especially after the 1990s, with a steady rise in the number of cases in the past seven years (Fig. 2). As depicted in the map, (Fig. 2) most of the new cases reported (11/20,) between 2010 and 2017, were from the Indian subcontinent, followed by China $(4 / 20)$. This emphasizes that possibility of primary laryngeal aspergillosis must be entertained in all cases, presenting with typical features of laryngeal inflammation along with hoarseness of voice.

Challenges in the diagnosis of laryngeal aspergillosis and utility of molecular diagnostics methods

As per the literature review, most of the laryngeal aspergillosis cases were diagnosed by the characteristic morphological features of the fungus in the biopsied material. 


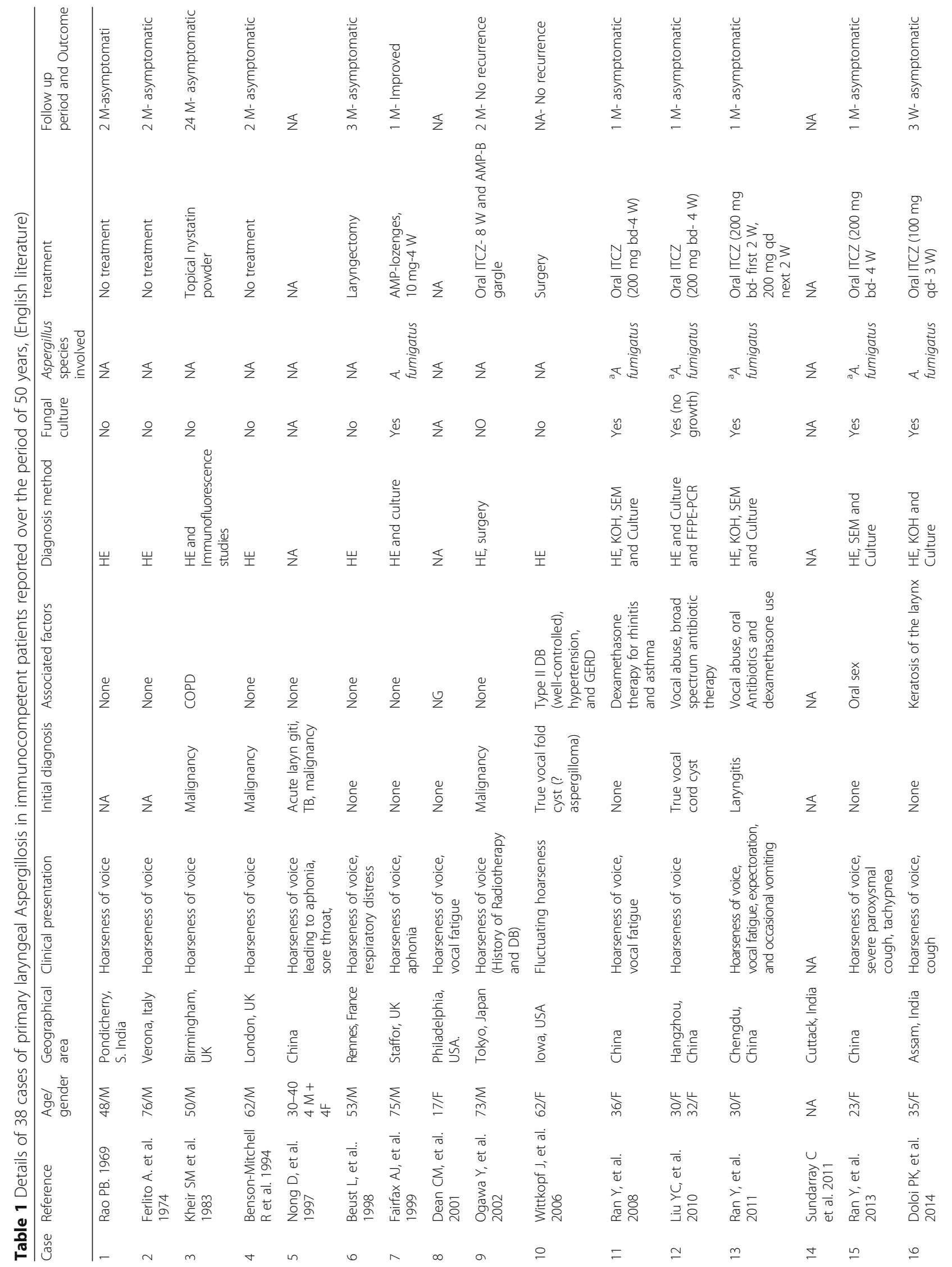




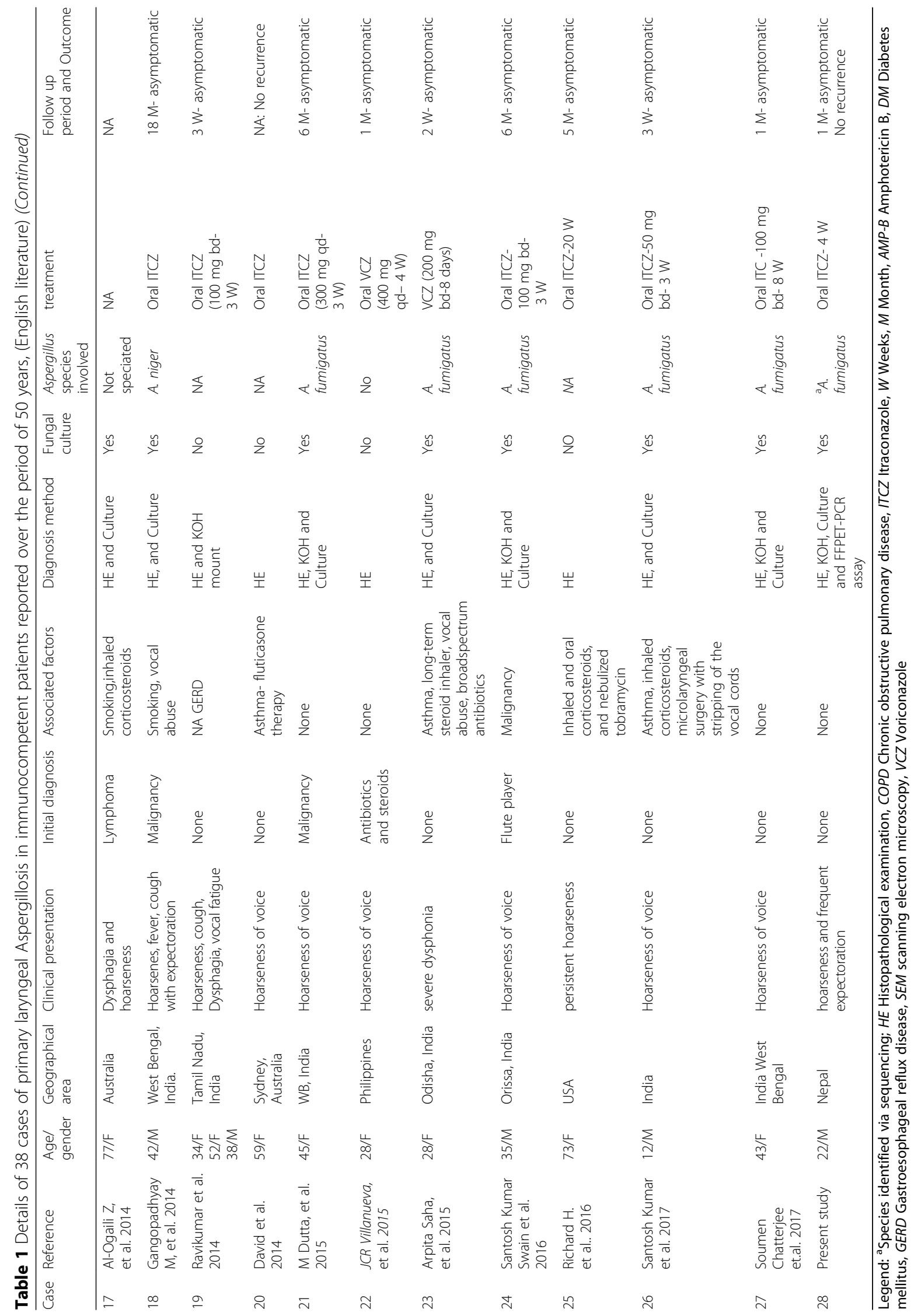




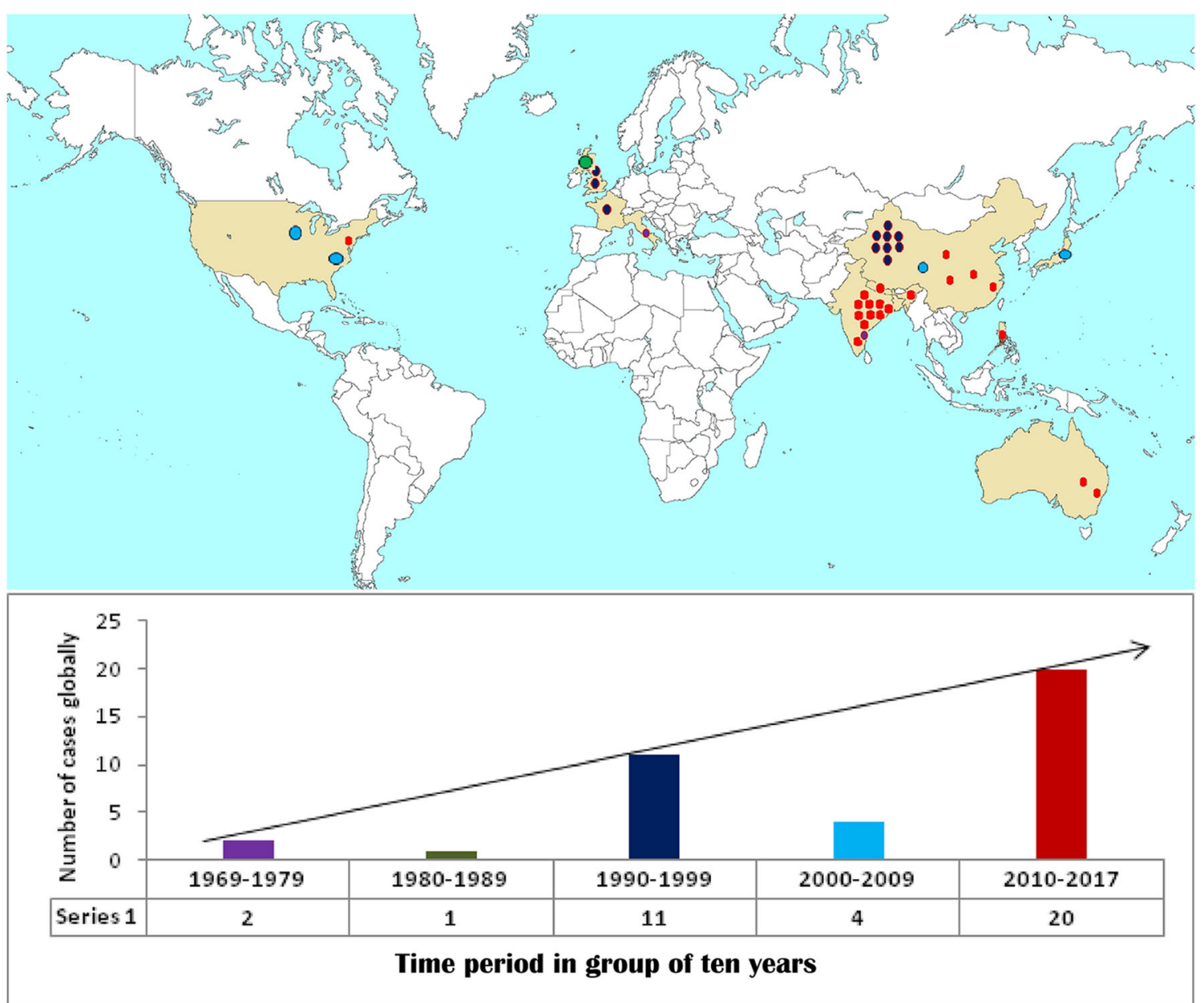

Fig. 2 Time trend and geographical distribution of 38 cases of primary laryngeal aspergillosis in immunocompetent patients. Legend: https://commons.wikimedia.org/wiki/Maps_of_the_world

However, result on species identification was lacking in majority of reported cases. Detection of hyphae, simulating those of Aspergillus in a biopsy specimen can be suggestive of fungal invasion but, is not necessarily pathognomonic of aspergillosis. Therefore, it becomes mandatory that the organism be isolated in pure culture and accurately identified. Few authors identified Aspergillus up to species level based on morphology and a couple of reports provided evidences of identification based on molecular methods $[11,12]$. Moreover it is well known that fungal identification with conventional culture technique has its own limitations. As per the studies [13] conducted earlier, as well as in the present case, we could not successfully culture the fungus from the laryngeal biopsy. In these situations, etiological identification directly from clinical specimen via extraction of DNA and sequencing is advantageous. In this study, PCR on DNA extracted from paraffin-embedded tissue confirmed the aetiology. The extended region of the gene encoding the large ribosomal subunit (28S) of fungi was used for PCR amplification and sequencing. This region was previously explored for designing of broad range PCR primers and showed generation of successful amplicons and sequences from yeasts and filamentous fungi [14]. Because of the paucity of sequences of this extended region of fungal $28 \mathrm{~S}$ rDNA in the public databases, the non D1/D2 region was rarely utilized for sequence-based detection and identification of fungi directly from clinical specimens. A recent study [15] showed the utility of the non D1/D2 region as a favorable target for the genus, and to a limited extent, species-level identification of pathogenic fungi in various fresh and FFPE samples. In the present study, attempt to amplify the internal transcribed spacer 1 (ITS1) region from the DNA extracted from the sample was not successful. One possible explanation might be due to the relatively larger size of the ITS1 region $(\sim 250-350 \mathrm{bp})$ than this non D1/D2 region $\left(198+\_25\right.$ bp). Although accurate species identification required sequencing of at least a partial ITS region such as ITS 1 or ITS2, the non D1/D2 multicopy gene could give a satisfying genus level 
identification. In our study, this region could identify the genus and species of the pathogen with clear discrimination from other species of Aspergilli (with less $\%$ similarity scores) as evidenced from the BLAST hits. Therefore, this non D1/D2 region must be considered for PCR-sequencing from direct clinical specimens in those cases where partial ITS genes fail to amplify.

\section{Treatment of cases}

In majority of the reported cases, including the present one, itraconazole was used as an empiric treatment, though voriconzole is the treatment of choice against invasive apsergillosis [16]. Possibly cost of the antifungal agent is an important limiting factor during treatment of fungal infections in developing countries. The critical condition of the patient, arising out of the acute laryngeal pathology may be a compelling reason for the empiric treatment on an emergency basis, yielding invariably positive outcome following therapy. Recent reports of the global emergence of azole resistance in A. fumigatus [17] may be of concern in the management of such patients in future. Prompt species identification and detection of resistance are of paramount importance in the management of laryngeal mycosis.

\section{Therapeutic outcome and relapse}

In all 38 cases reviewed (Table 1), there was complete resolution of symptoms without any relapse, irrespective of the therapeutic modality adopted. There was not much difference in the time period between administration of antifungal drugs and relief of symptoms, regardless of whether the drug administered was itraconazole or voriconazole. Thus, considering the toxicity of conventional amphotericin B, and the cost of liposomal amphotericin B; empiric therapy with either itraconazole or voriconazole may be strongly advocated as better therapeutic options.

\section{Conclusion}

Since last few decades cases of primary laryngeal aspergillosis in immunocompetent individuals are on the rise, globally. Patients responded to azoles with good prognosis. This is the first case of invasive laryngeal mycosis reported in Nepal. The extraction of DNA from tissue and sequencing helps to identify the etiological agent, when culture fails to isolate the fungus.

\footnotetext{
Abbreviations

AMP-B: Amphotericin B; ATCC: American Type Culture Collection; COPD: Chronic obstructive pulmonary disease; DM: Diabetes mellitus; DNA: Deoxyribonucleic acid; FFPET: Formalin fixed paraffin embedded tissue; GERD: Gastroesophageal reflux disease; HE: Histopathological examination; HIV: Human immunodeficiency virus; ITCZ: Itraconazole; ITS: Internal transcribed spacer; PCR: Polymerase chain reaction; SEM: Scanning electron microscopy; VCZ: Voriconazole; VDRL: Venereal disease research laboratory test
}

\section{Acknowledgements}

The authors gratefully acknowledge the Dr. Narasimha Rao, professor of pathology for expertise opinion and Dr. Shishir Gokhale, professor of microbiology, Dr. Deependra Hamal, faculty of microbiology, Manipal College of Medical Sciences, Nepal for support in drafting the manuscript. We also extended special thanks to Dr. Peralam Yegneswaran Prakash, In-charge medical mycology laboratory, Kasturba Medical College and Dr. Indira Bairy, Professor and Head, Department of Microbiology, Melaka Manipal Medical College, Manipal University, India for valuable suggestions. We extend our special thanks to Manipal Teaching Hospital, Pokhara, Nepal, for providing the facility to carry out the study.

\section{Availability of data and materials}

All data generated or analyzed during this study are included in this published article. The sequence data have been deposited in the GenBank database (http://www.ncbi.nlm.nih.gov/Genbank/index.html) with the accession number MH465665.

\section{Authors' contributions}

SHS: observed the incidence and case, performed the laboratory tests, followed the case, literature review and wrote the manuscript; KR: observed the incidence and case, collected specimens, case follow-up, and treatment, JJ: molecular studies and manuscript preparation, SMR: molecular analysis and manuscript drafting, NN \& AC: Contributed toward providing clinical relevance, manuscript drafting, and critically reviewed the manuscript, AG: contributed toward histopathological diagnosis. All authors read and approved the final manuscript.

\section{Ethics approval and consent to participate}

Due approval from the Institutional Research and Ethics Committee of the Manipal College of Medical Sciences, Pokhara, Nepal was obtained for analysing the case study, for writing the manuscript. Consent was obtained from patient to publish the data.

\section{Consent for publication}

Authors would like to acknowledge the patient, who gave written, informed consent for the publication of this case report.

\section{Competing interests}

The authors declare that they have no competing interests.

\section{Publisher's Note}

Springer Nature remains neutral with regard to jurisdictional claims in published maps and institutional affiliations.

\section{Author details}

${ }^{1}$ Department of Medical Microbiology, Manipal College of Medical Sciences, Pokhara, Nepal. ${ }^{2}$ Department of Medical Microbiology, Postgraduate Institute of Medical Education and Research, Chandigarh, India.

Received: 10 December 2017 Accepted: 26 June 2018

Published online: 11 July 2018

\section{References}

1. Athanassiadou F, Kourti M, Papageorgiou T, Danielidis J. Invasive aspergillosis of the larynx in a child with acute lymphoblastic leukemia. Pediatr Infect Dis J. 2005;24:190-1.

2. Vrabec DP. Fungal infections of the larynx. Otolaryngol Clin N Am. 1993; 26(6):1091-114.

3. Dutta M, Jotdar A, Kundu S, Ghosh B, Mukhopadhyay S. Primary laryngeal aspergillosis in the immunocompetent state: a clinical update. Braz J Otorhinolaryngol. 2017;83(2):228-34. https://doi.org/10.1016/j.bjorl.2015.06.002.

4. Rao PB. Aspergillosis of larynx. J Laryngol Otol. 1969;83(4):377-9.

5. Lau A, Chen S, Sorrell T, Carter D, Malik R, Martin P, Halliday C. Development and clinical application of a panfungal PCR assay to detect and identify fungal DNA in tissue specimens. J Clin Microbiol. 2007;45(2):380-5.

6. Gangopadhyay M, Majumdar K, Bandyopadhyay A, Ghosh A. Invasive primary aspergillosis of the larynx presenting as hoarseness and a chronic nonhealing laryngeal ulcer in an immunocompetent host: a rare entity. Ear Nose Throat J. 2014;93:265-8. 
7. Gallo A, Manciocco V, Simonelli M, Minni A, De Vincentiis M. Clinical findings of laryngeal aspergillosis. Otolaryngol Head Neck Surg. 2000;123(5):661-2.

8. Barnes RA, Rogers TR. Aspergillosis in immunocompromised patients part I: the problem of diagnosis. In: Drouhet E, Cole GT, de Repentigny L, Latgé JP, Dupont B, editors. Fungal Antigens. Boston: Springer; 1988.

9. Vennewald I, Henker M, Klemm E, Seebacher C. Fungal colonization of the paranasal sinuses. Mycoses. 1999;42(Suppl 2):33-6.

10 Darley D, Lowinger D, Plit M. Laryngeal aspergilloma: a complication of inhaled fluticasone therapy for asthma. Respirology Case Reports. 2014;2(4): 123-5. https://doi.org/10.1002/rcr2.70.

11. Ran Y, Yang B, Liu S, Dai Y, Pang Z, Fan J, Bai H, Liu S. Primary vocal cord aspergillosis caused by aspergillus fumigatus and molecular identification of the isolate. Med Mycol. 2008 Aug;46(5):475-9. https://doi.org/10.1080/ 13693780701851703

12. Ran Y, Li L, Cao L, Dai Y, Wei B, Zhao Y, Liu Y, Bai H, Zhang C. Primary vocal cord aspergillosis and scanning electron microscopical observation of the focus of infection. Mycoses. 2011;54(5):e634-7. https://doi.org/10.1111/j. 1439-0507.2010.01954.x.

13. Liu YC, Zhou SH, Ling L. Aetiological factors contributing to the development of primary laryngeal aspergillosis in immunocompetent patients. J Med Microbiol. 2010;59:1250-3. https://doi.org/10.1099/jmm.0.021634-0.

14. Khot PD, Ko DL, Fredricks DN. Sequencing and analysis of fungal rRNA operons for development of broad-range fungal PCR assays. Appl Environ Microbiol. 2009;75(6):1559-65. https://doi.org/10.1128/AEM.02383-08.

15. Gade L, Hurst S, Balajee SA, Lockhart SR, Litvintseva AP. Detection of mucormycetes and other pathogenic fungi in formalin fixed paraffin embedded and fresh tissues using the extended region of $28 \mathrm{~S}$ rDNA. Med Mycol. 2017;55(4):385-95. https://doi.org/10.1093/mmy/myw083.

16. Patterson TF, Thompson GR, Denning DW, et al. Practice guidelines for the diagnosis and Management of Aspergillosis: 2016 update by the Infectious Diseases Society of America. Clin Infect Dis. 2016;63(4):e1-60.

17. Jacques F. Meis, Anuradha Chowdhary, Johanna L. Rhodes, Matthew C. Fisher, Paul E. Verweij. Clinical implications of globally emerging azole resistance in Aspergillus fumigatus. Phil Trans R Soc B. 2016;371:20150460. https://doi.org/10.1098/rstb.2015.0460. Published 24 October 2016.

\section{Ready to submit your research? Choose BMC and benefit from:}

- fast, convenient online submission

- thorough peer review by experienced researchers in your field

- rapid publication on acceptance

- support for research data, including large and complex data types

- gold Open Access which fosters wider collaboration and increased citations - maximum visibility for your research: over $100 \mathrm{M}$ website views per year

At BMC, research is always in progress.

Learn more biomedcentral.com/submissions 